\title{
Evaluación de niveles de energía digestible en dos sistemas de alimentación en la respuesta productiva y reproductiva de cuyes (Cavia porcellus)
}

\author{
Assessment of digestible energy levels in two feeding systems in the productive \\ and reproduction performance of guinea pigs (Cavia porcellus)
José Antonio Sarria Bardales ${ }^{1}$, Víctor Vergara Rubín ${ }^{2}$, Jose Luis Cantaro Segura ${ }^{1,4}$, Pedro Alejandro Rojas ${ }^{1}$

\section{Resumen}

\begin{abstract}
El objetivo del presente estudio fue comparar dietas con dos niveles de energía digestible (2.7 y 2.9 Mcal de ED/kg) y dos sistemas de alimentación (con o sin la inclusión de forraje verde) para cuyes hembras reproductoras, evaluando la respuesta en parámetros productivos y reproductivos (ganancia de peso, consumo de alimento, tamaño de camada, porcentaje de fertilidad, partos, abortos, mortalidad y pesos promedios al nacimiento y destete), así como la respuesta económica. Se trabajó con 60 hembras de segundo parto de $1274 \mathrm{~g}$ de peso vivo y 12 cuyes machos mejorados, procedentes de la Granja de Cuyes de Cieneguilla de la Universidad Nacional Agraria La Molina, Lima, Perú. El estudio tuvo una duración de 18 semanas entre mayo y octubre de 2010. Se utilizó un diseño estadístico factorial de $2 \times 2$ con cuatro tratamientos y tres repeticiones por tratamiento: T1 y T2 con dietas de $2.7 \mathrm{Mcal}$ de ED $/ \mathrm{kg}$ con y sin inclusión de forraje verde, respectivamente, y el T3 y T4 con dietas de $2.9 \mathrm{Mcal}$ de ED $/ \mathrm{kg}$ con y sin inclusión de forraje verde, respectivamente. No se encontraron diferencias significativas entre tratamientos en la mayoría de los indicadores; sin embargo, las madres alimentadas bajo sistemas de inclusión de forraje perdieron peso al destete $(-47.6 \mathrm{~g})$, mientras que dietas con el nivel de energía 2.9 Mcal de ED/kg produjeron mayor peso al parto $(\mathrm{p}<0.05)$. El menor costo por cría destetada se obtuvo bajo el sistema de alimentación con inclusión de forraje (S/. $5.00 \mathrm{vs.} \mathrm{S/.5.60).}$
\end{abstract}

Palabras clave: cuyes; energía; balanceado; forraje; reproductoras

\footnotetext{
${ }^{1}$ Departamento de Producción Animal, Facultad de Zootecnia, Universidad Nacional Agraria La Molina, Lima, Perú

${ }^{2}$ Departamento de Nutrición, Facultad de Zootecnia, Universidad Nacional Agraria La Molina, Lima, Perú

${ }^{4}$ E-mail: jcantaro@lamolina.edu.pe
}

Recibido: 18 de febrero de 2019

Aceptado para publicación: 30 de octubre de 2019 
The aim of this study was to compare diets with two levels of digestible energy (2.7 and 2.9 Mcal of ED/kg) and two feeding systems (with or without the inclusion of green forage) in female breeding guinea pigs, evaluating the response in productive and reproductive parameters (weight gain, food intake, litter size, fertility rate, births, abortions, mortality and birth and weaning weights), as well as the economic response. The study was conducted with 60 second-parity females of $1274 \mathrm{~g}$ of mean bodyweight and 12 improved male guinea pigs from the Cieneguilla Guinea Pig Farm of the La Molina Agrarian University, Lima, Peru. The study lasted for 18 weeks between May and October 2010. A $2 \times 2$ factorial statistical design was used with four treatments and three repetitions per treatment: T1 and T2 with diets of $2.7 \mathrm{Mcal}$ of ED $/ \mathrm{kg}$ with and without inclusion of green forage, respectively, and T3 and T4 with diets of $2.9 \mathrm{Mcal}$ of ED/kg with and without inclusion of green forage, respectively. No significant differences were found between treatments in most of the indicators; however, mothers fed under forage inclusion systems lost weight at weaning (-47.6 g), while diets with the 2.9 Mcal energy level of ED $/ \mathrm{kg}$ produced greater birth weight $(\mathrm{p}<0.05)$. The lowest cost per weaned offspring was obtained under the feed system including forage (S/. 5.00 vs. S/. 5.60).

Key words: guinea pigs; energy; concentrate; forage; breeding animals

\section{INTRODUCCIÓN}

En el Perú hay un gran interés por la crianza del cuy (Cavia porcellus), no solo por ser una valiosa fuente de nutrientes para el poblador rural, sino también como una fuente de ingreso económico, lo cual ha fomentado la búsqueda todos de genotipos y sistemas de alimentación que permitan satisfacer las expectativas de los criadores (Camino e Hidalgo, 2014).

La mayor parte de trabajos existentes sobre requerimientos nutricionales del cuy se han hecho considerando al cuy como animal de laboratorio y no como animal de producción cárnica. No obstante, Torres et al. (2006) evaluaron dos niveles de ED (2.9 y $3.0 \mathrm{Mcal})$ y proteína (15 y 18\%), obteniendo los mejores rendimientos con los animales que consumieron dietas con $18 \%$ de proteína; mientras que Remigio (2006) en un estudio con varios niveles de lisina y aminoácidos azufrados en dietas isoenergéticas encontró los mejores rendimientos al utilizar niveles entre 0.78 a $0.84 \%$ de lisina y de 0.71 a $0.79 \%$ de aminoácidos azufrados. Según el NRC (1978), los requerimientos de energía para cuyes son de 3.0 Mcal ED/kg; no obstante, resultados de investigación en el Perú señalan que para las etapas de crecimiento, engorde, gestación y lactación serían adecuados $2.90,2.86$ y $2.86 \mathrm{Mcal} \mathrm{ED} / \mathrm{kg}$, respectivamente (Airahuacho y Vergara, 2017).

La introducción de alimento balanceado en la crianza de cuyes genéticamente mejorados se ha venido desarrollando en la búsqueda por atender los requerimientos nutricionales de esta especie en sus diversas etapas productivas. Investigaciones en etapas de inicio y crecimiento demuestran la respuesta positiva de los cuyes al sistema de alimentación sin inclusión de forraje. Condori (2014) demostró que existe una mayor retribución económica en cuyes alimentados con dietas sin inclusión de forraje respecto a dietas con inclusión; además, considerando el factor energía digestible, Airahuacho y Vergara (2017) encontraron un efecto favorable sobre la ganancia de peso, consumo de 
alimento, conversión alimenticia y rendimiento de carcasa con dietas de 2.9 Mcal de ED $/ \mathrm{kg}$ de alimento. Por otro parte, Morales et al. (2011) reportaron que el mayor nivel energético nutricional de la dieta ocasiona un menor consumo de alimento y por lo mismo una mejor conversión alimenticia; sin embargo, en la etapa de reproducción no se dispone de suficiente información sobre el efecto de los sistemas de alimentación alternativos que se usan en campo.

El objetivo del presente estudio fue evaluar el efecto de dos niveles de energía empleados en dos sistemas de alimentación durante la etapa de reproducción de cuyes, midiendo los resultados a través de parámetros productivos y reproductivos, además de una evaluación económica relativa al costo de alimentación de cada tratamiento.

\section{Materiales y Métodos}

El estudio se llevó a cabo en las instalaciones de la Granja de Animales Menores del Programa de Investigación y Proyección Social de Aves y Animales Menores de la Facultad de Zootecnia de la Universidad Nacional Agraria La Molina (UNALM), en Lima, Perú. Las dietas experimentales y el forraje verde fueron analizados en el Laboratorio de Evaluación Nutricional de Alimentos (LENA) de la misma institución.

Se utilizaron 60 cuyes hembras mejoradas Tipo 1 de segundo parto, con peso promedio de $1274 \mathrm{~g}$, y 12 cuyes machos de $1516 \mathrm{~g}$ de peso promedio, todos provenientes de la Granja de Cuyes de Cieneguilla de la UNALM. Los animales fueron distribuidos al azar en 12 unidades experimentales (cinco hembras y un macho) en pozas de cemento a nivel del piso con $1.8 \mathrm{~m}^{2}$ por cada unidad experimental, entre mayo y octubre de 2010.

Los tratamientos fueron: $\mathrm{T} 1$ y $\mathrm{T} 2,2.7$ Mcal de energía digestible/kg de alimento balanceado con y sin inclusión de forraje ver- de, respectivamente; T3 y T4, 2.9 Mcal de energía digestible/kg de alimento balanceado con y sin inclusión de forraje verde. El suministro de alimento y de agua fue ad libitum.

Las dietas fueron formuladas, preparadas y peletizadas en la Planta de Alimentos Balanceados del Programa de Investigación y Proyección Social en Alimentos, UNALM (Cuadro 1). Para el T2 y T4 bajo sistema de alimentación sin inclusión de forraje verde se incluyó vitamina $\mathrm{C}$ en la forma poli fosfatada (Rovimix Stay 35®) a fin de cubrir los requerimientos del nutriente en esta especie. En T1 y T3 se suministró maíz chala (Zea mays) de manera diaria y restringida $(10 \%$ del peso vivo/animal/día). Este forraje fue cortado a los 60 días de crecimiento y provino de siembras de la UNALM. Los cuatro tratamientos fueron formulados con un nivel de fibra de $10 \%$.

Las dietas experimentales y el forraje verde fueron sometidos a análisis proximal (Cuadros 2 y 3 ) para determinar materia seca, humedad, proteína cruda (nitrógeno total), fibra cruda, lípidos totales, ceniza y extracto libre de nitrógeno, utilizando las técnicas establecidas por la A.O.A.C. (2005).

Los parámetros productivos evaluados fueron el peso promedio (g) al empadre, parto, destete y la variación de peso parto-destete, además del peso promedio de las crías (g) al nacimiento y destete. Así mismo, el consumo de alimentos (diferencia entre lo ofrecido y el residuo de cada semana). Todos los pesos fueron registrados utilizando una balanza de mesa digital MIRAY de $3 \mathrm{~kg}$ de capacidad con precisión de $0.1 \mathrm{~g}$. En los parámetros reproductivos se evaluó (a) porcentaje de fertilidad (relación entre el número de hembras gestantes y las hembras empadradas), (b) porcentaje de abortos (relación entre el número de hembras abortadas y las hembras gestantes), (c) porcentaje de natalidad (relación entre el número de hembras paridas y las hembras gestantes), (d) tamaño de camada total al nacimiento (rela- 
Cuadro 1. Fórmula de las dietas experimentales para cuyes y su valor nutritivo calculado (expresado en porcentaje)

\begin{tabular}{lcccc}
\hline Composición & \multicolumn{4}{c}{ Tratamientos } \\
\cline { 2 - 5 } & $\mathrm{T} 1$ & $\mathrm{~T} 2$ & $\mathrm{~T} 3$ & $\mathrm{~T} 4$ \\
\hline Subproducto de trigo & 66.28 & 66.25 & 35.25 & 35.22 \\
Mezcla de cereales & 12.00 & 12.00 & 17.00 & 17.00 \\
Torta de soya, 47 & 11.00 & 11.00 & 21.00 & 21.00 \\
Maíz amarillo & 8.00 & 8.00 & 23.00 & 23.00 \\
Aceite vegetal & - & - & 1.00 & 1.00 \\
Carbonato de calcio & 1.80 & 1.80 & 1.80 & 1.80 \\
Sal & 0.38 & 0.38 & 0.40 & 0.40 \\
Cloruro de colina, 60 & 0.20 & 0.20 & 0.20 & 0.20 \\
Inhibidor de hongos & 0.15 & 0.15 & 0.15 & 0.15 \\
Premezcla Vit + Min. & 0.14 & 14.00 & 0.14 & 0.14 \\
Rovimix 35 (Vit. C) & - & 0.03 & - & 0.03 \\
DL-Metionina & 0.05 & 0.05 & 0.06 & 0.06 \\
\hline Total & 100.0 & 100.0 & 100.0 & 100.0 \\
\hline Contenido nutricional & & & & \\
$\quad$ Energía digestible (Mcal/kg) & 2.70 & 2.70 & 2.90 & 2.90 \\
$\quad$ Proteína cruda (\%) & 17.00 & 17.00 & 19.00 & 19.00 \\
Fibra cruda (\%) & 10.30 & 10.30 & 10.00 & 10.00 \\
$\quad$ Lisina (\%) & 0.80 & 0.80 & 0.95 & 0.95 \\
Metionina-cistina (\%) & 0.62 & 0.62 & 0.65 & 0.65 \\
Arginina (\%) & 1.21 & 1.22 & 1.31 & 1.31 \\
Treonina (\%) & 0.60 & 0.60 & 0.70 & 0.70 \\
Triptófano (\%) & 0.29 & 0.29 & 0.29 & 0.29 \\
Sodio (\%) & 0.08 & 0.08 & 0.09 & 0.09 \\
Potasio (\%) & 0.82 & 0.85 & 0.84 & 0.84 \\
Calcio (\%) & 0.81 & 0.82 & 0.76 & 0.76 \\
FDN (\%) & 36.10 & 36.14 & 29.37 & 29.37 \\
\hline
\end{tabular}

Fuente: Planta de Alimentos del Programa de Investigación y Proyección Social en Alimentos de la Facultad de Zootecnia, UNALM

T1 y T2, 2.7 Mcal de energía digestible/kg de alimento balanceado con y sin inclusión de forraje verde, respectivamente; T3 y T4, 2.9 Mcal de energía digestible/kg de alimento balanceado con y sin inclusión de forraje verde

ción entre el número total de crías nacidas y las hembras gestantes paridas), (e) tamaño de camada nacida viva al nacimiento (relación entre el número total de crías nacidas vivas y las hembras gestantes paridas), y (f) tamaño de camada promedio al destete (relación entre el número total crías destetadas de 14 días y las hembras gestantes paridas).
Se evaluó, además, la retribución y el mérito económico relativo de los tratamientos, considerando solo el costo de alimentación por reproductora (Car), cuyo resultado es el producto de la cantidad total de alimento consumido en tal como ofrecido (TCO) durante la etapa experimental (balanceado y forraje verde) por el precio de cada dieta, 
Cuadro 2. Análisis químico proximal de las dietas experimentales (tal como ofrecido)

\begin{tabular}{lcccc}
\hline \multirow{2}{*}{ Nutrientes $(\%)$} & T1 & T2 & T3 & T4 \\
\cline { 2 - 5 } & 10.60 & 10.70 & 11.60 & 11.00 \\
Humedad & 89.40 & 89.30 & 88.40 & 89.00 \\
Materia seca & 17.50 & 17.80 & 19.10 & 18.80 \\
Proteína & 2.85 & 2.90 & 3.00 & 2.88 \\
Grasa & 10.10 & 10.30 & 9.98 & 10.08 \\
Fibra & 6.80 & 7.60 & 8.20 & 8.05 \\
Ceniza & 52.15 & 50.70 & 48.12 & 49.19 \\
ELN &
\end{tabular}

Fuente: Laboratorio de Evaluación Nutricional de Alimentos (LENA) del Departamento Académico de Nutrición de la Facultad de Zootecnia, UNALM

T1 y T2, 2.7 Mcal de energía digestible/kg de alimento balanceado con y sin inclusión de forraje verde, respectivamente; T3 y T4, 2.9 Mcal de energía digestible/kg de alimento balanceado con y sin inclusión de forraje verde

Cuadro 3. Análisis químico proximal del forraje verde (maíz chala)

\begin{tabular}{lcc}
\hline Componente & $\begin{array}{c}\text { Tal como } \\
\text { ofrecido }\end{array}$ & $\begin{array}{c}\text { Base } \\
\text { seca }\end{array}$ \\
\hline Humedad (\%) & 83.30 & 0 \\
Materia seca (\%) & 16.70 & 100.0 \\
Proteína total (N x & 1.67 & 10.00 \\
6.25) \% & & 1.98 \\
Extracto etéreo (\%) & 0.33 & 28.02 \\
Fibra cruda (\%) & 4.68 & 8.80 \\
Ceniza (\%) & 1.47 & 51.20 \\
ELN (\%) & 8.55 & \\
\hline
\end{tabular}

Fuente: Laboratorio de Evaluación Nutricional de Alimentos (LENA) del Departamento Académico de Nutrición de la Facultad de Zootecnia, UNALM

según el tratamiento. Para el precio del alimento balanceado se consideró un adicional de $10 \%$ sobre el costo del alimento en planta. Dicho porcentaje considera gastos de transporte y comercialización de las raciones, simulando el precio comercial del alimento. El costo de alimentación por cuy destetado
(Cacd) se obtuvo mediante el cociente del costo de alimentación por reproductora (Car) y el tamaño de camada al destete (Tcd).

Para el análisis de los resultados se optó por el modelo de Diseño Completamente al Azar con arreglo factorial de dos factores (2x2), el cual evaluó la influencia de dos niveles de energía digestible $(2.7$ y $2.9 \mathrm{Mcal} / \mathrm{kg}$ ) respecto a los dos sistemas de alimentación (con o sin inclusión de forraje), dando como resultado los efectos individuales y la interacción entre ambas variables. Se empleó el análisis de variancia para determinar las diferencias significativas de los tratamientos $(\alpha=0.05)$ y la prueba de Duncan para determinar diferencias entre las medias de los tratamientos. Todos los cálculos estadísticos se desarrollaron con el uso del software SAS System v. 8.0.

\section{Resultados y Discusión}

Los resultados demuestran que los niveles de energía digestible en dietas con o sin inclusión de forraje verde no afectaron significativamente los parámetros de fertili- 
dad, abortos, natalidad, tamaño de camada al nacimiento y al destete; así como tampoco los parámetros de peso de las madres al empadre, parto, destete y variación de peso parto-destete (Cuadro 4). El peso promedio general de empadre fue similar al reportado por Chauca et al (2008), considerando 1282.3 $\pm 200 \mathrm{~g}$ como peso de empadre para hembras de segundo parto.

El efecto del nivel de energía para el parámetro de peso al parto fue estadísticamente significativo $(\mathrm{p}<0.05)$, obteniéndose un incremento de hasta $5 \%$ del peso final al utilizar 2.9 Mcal $/ \mathrm{kg}$ de ED en el alimento. Peruano (1999) evaluó la vida reproductiva del cuy hembra en cuatro partos bajo alimentación mixta reportando como peso promedio al parto $1354 \mathrm{~g}$; sin embargo, bajo el mismo sistema de alimentación, el presente estudio obtuvo pesos al parto de $1800 \mathrm{~g}$, debiendo considerarse en este sentido, al margen del avance genético, que en el presente estudio se hizo uso del sistema de empadre controlado.

El sistema de alimentación no presentó diferencias significativas entre tratamientos. Pedraz (2001), al evaluar hembras mejoradas de primer parto procedentes de Lima, Arequipa y Cajamarca, teniendo como testigo, reproductoras de la UNALM, bajo un sistema de alimentación con inclusión de forraje verde, determinó que las hembras procedentes de Arequipa mostraron mayores pesos al parto (1425.2 g) seguidas por el tratamiento testigo (1335.8 g). Así mismo, Revilla (2011) utilizando dietas isoenergéticas de 2.9 Mcal de ED/kg bajo alimentación integral obtuvo $1488 \mathrm{~g}$ como peso promedio general al parto.

Los resultados obtenidos concuerdan con Solórzano (2014), quien tampoco encuentra diferencias significativas para el peso de reproductoras al parto, presentándose valores numéricos más altos en sus tratamientos integrales (1309 g), al igual que en el presente estudio donde con hembras de segundo parto en el sistema de alimentación sin inclu- sión de forraje verde se logró 1839 g. Por otro lado, el efecto aislado de los sistemas de alimentación presentó diferencias significativas $(\mathrm{p}<0.05)$, donde la variación del peso parto-destete en la dieta sin inclusión de forraje mostró una ganancia de peso de $24.2 \mathrm{~g}$, probablemente debido a que el alimento integral hace un mejor aporte de los requerimientos nutricionales necesarios que contribuyen a cubrir la demanda de energía y demás nutrientes que presentan las reproductoras, los cuales no son posibles de cubrir con la ingesta exclusiva o adicional de forraje verde.

En general, el T4 mostró la mejor tendencia productiva respecto al resto. Peruano (1999) reportó pérdidas de peso entre el parto $y$ el destete de $-2.84 \mathrm{~g}$ bajo un sistema con inclusión de forraje en hembras de segundo parto, mientras que en el presente estudio se tuvo pérdidas de $-47.6 \mathrm{~g}$ bajo el mismo sistema de alimentación. No obstante, Solórzano (2014) evaluó tres sistemas de alimentación comercial en reproductoras de los genotipos Allin Perú y Cieneguilla no encontrando diferencias entre sus tratamientos, con incrementos de $14.9 \mathrm{~g}$ para alimento de granja en harina con $3.0 \mathrm{Mcal}$ de ED/kg y 14.8 g para alimento comercial en pellet.

Respecto a los consumos promedios de alimento en MS, no hubo diferencia significativa entre tratamientos (Cuadro 5); sin embargo, se presentó un incremento en la ingesta de alimento de hasta $24 \%$ en los sistemas de alimentación con inclusión de forraje. Al evaluar el factor aislado de los sistemas alimenticios se evidencia mayor consumo para las dietas con inclusión de forraje $(p<0.01)$. El consumo más alto se dio en el T3 y el más bajo en el T2, posiblemente debido a que el cuy, al ser un animal herbívoro, manifiesta preferencias por el forraje verde. No obstante, Sarmiento (2014), a pesar de las diferencias y la gradiente numérica, no encontró diferencias estadísticas entre sus tratamientos, usando como testigo una dieta mixta con $105.2 \mathrm{~g}$ de consumo promedio de MS. El carácter materno de las reproductoras hace que los consumos se incrementen para 
Cuadro 4. Efecto de los sistemas de alimentación y nivel de energía sobre la variación de peso en cuyes reproductoras

\begin{tabular}{|c|c|c|c|c|c|c|}
\hline 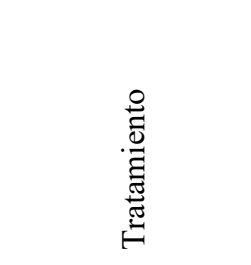 & 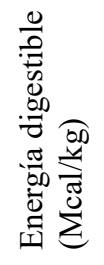 & 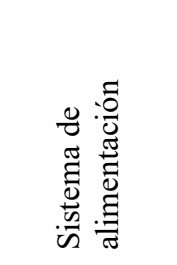 & 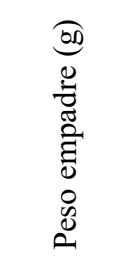 & $\begin{array}{l}\text { क्रि } \\
0 \\
0 \\
0 \\
0 \\
0 \\
0 \\
0 \\
0\end{array}$ & 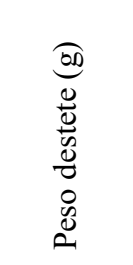 & 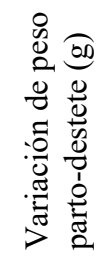 \\
\hline $\mathrm{T}-1$ & 2.7 & C/inclusión & $1,315.2^{\mathrm{a}}$ & $1,778.3^{\mathrm{a}}$ & $1,727.8^{\mathrm{a}}$ & $-56.5^{\mathrm{a}}$ \\
\hline $\mathrm{T}-2$ & 2.7 & S/inclusión & $1,270.4^{\mathrm{a}}$ & $1,760.0^{\mathrm{a}}$ & $1,782.0^{\mathrm{a}}$ & $22.0^{\mathrm{a}}$ \\
\hline $\mathrm{T}-3$ & 2.9 & C/inclusión & $1,285.5^{\mathrm{a}}$ & $1,821.6^{\mathrm{a}}$ & $1,777.0^{\mathrm{a}}$ & $-44.6^{\mathrm{a}}$ \\
\hline $\mathrm{T}-4$ & 2.9 & S/inclusión & $1,250.3^{\mathrm{a}}$ & $1,918.1^{\mathrm{a}}$ & $1,944.4^{\mathrm{a}}$ & $26.3^{\mathrm{a}}$ \\
\hline Efecto del nivel & 2.7 & & $1,292.8^{\mathrm{a}}$ & $1,769.2^{b}$ & $1,754.9^{\mathrm{a}}$ & $-14.2^{\mathrm{a}}$ \\
\hline de energía & 2.9 & & $1,267.9^{\mathrm{a}}$ & $1,869.8^{\mathrm{a}}$ & $1,860.7^{\mathrm{a}}$ & $-9.1^{\mathrm{a}}$ \\
\hline Efecto del & & C/inclusión & $1,300.4^{\mathrm{a}}$ & $1,800.0^{\mathrm{a}}$ & $1,752.4^{\mathrm{a}}$ & $-47.6^{b}$ \\
\hline $\begin{array}{l}\text { sistema de } \\
\text { alimentación }\end{array}$ & & S/inclusión & $1,260.4^{\mathrm{a}}$ & $1,839.1^{\mathrm{a}}$ & $1,863.2^{\mathrm{a}}$ & $24.2^{\mathrm{a}}$ \\
\hline & & & \multicolumn{4}{|c|}{ Probabilidad } \\
\hline \multicolumn{3}{|l|}{ Energía } & 0.306 & 0.027 & 0.067 & 0.872 \\
\hline \multicolumn{3}{|l|}{ Sistema } & 0.117 & 0.324 & 0.057 & 0.047 \\
\hline \multicolumn{3}{|l|}{ Energía*Sistema } & 0.839 & 0.161 & 0.289 & 0.980 \\
\hline
\end{tabular}

tratar de cubrir sus requerimientos nutricionales. Por otro lado, al evaluar independientemente el nivel de energía se encontraron valores muy cercanos; es decir, de 107.5 g/animal/día para dietas con $2.7 \mathrm{Mcal}$ de $\mathrm{ED} / \mathrm{kg}$ y 110.9 para dietas con $2.9 \mathrm{Mcal}$ de $\mathrm{ED} / \mathrm{kg}$.

En resumen, los consumos de MS más bajos, aunque sin diferencias estadísticas entre sí, se dieron en dietas sin inclusión de forraje, ya que estas dietas brindan, por mayor densidad de nutrientes, los aportes necesarios para mantener adecuadamente la gestación y la lactancia. Estos resultados concuerdan con Solórzano (2014), quien presenta consumos de $90.9 \mathrm{~g} /$ día para dietas comercial en pellet con inclusión de forraje y con- sumos de $87.6 \mathrm{~g} /$ día para dietas sin inclusión, valores similares al presente estudio. Además, Torres et al. (2006) reportaron diferencias de consumo en materia seca en cuyes que recibieron dietas de 2.8 y $3.0 \mathrm{Mcal} \mathrm{ED} / \mathrm{kg}$ y $18 \%$ de PB bajo sistema de alimentación mixta; sin embargo, Morales et al. (2011), utilizando cuyes de la línea Perú, no encontraron diferencias de consumo en materia seca en animales alimentados con dietas isonutritivas de 2.8 y $3.0 \mathrm{Mcal} \mathrm{ED} / \mathrm{kg}$. Revilla (2011), por su parte, obtuvo consumos de MS en cuyes mejorados sin inclusión de forraje de 84.4 a $97.8 \mathrm{~g} /$ día, concluyendo que dietas con inclusión de forraje tienen mayor consumo de MS, debido a la menor concentración de nutrientes respecto a la ofrecida en sistemas sin inclusión de forraje. 
Cuadro 5. Efecto de los sistemas de alimentación y nivel de energía sobre el consumo en cuyes reproductoras

\begin{tabular}{|c|c|c|c|c|c|c|c|c|c|}
\hline \multirow{2}{*}{\multicolumn{2}{|c|}{ Consumo de alimento }} & \multicolumn{4}{|c|}{ Tratamientos } & \multicolumn{2}{|c|}{$\begin{array}{l}\text { Sistema de } \\
\text { alimentación }\end{array}$} & \multicolumn{2}{|c|}{$\begin{array}{l}\text { Nivel de energía } \\
\text { (Mcal ED/kg de } \\
\text { alimento) }\end{array}$} \\
\hline & & $\mathrm{T} 1$ & $\mathrm{~T} 2$ & $\mathrm{~T} 3$ & $\mathrm{~T} 4$ & $\begin{array}{c}\text { Con } \\
\text { inclusión } \\
\text { de forraje }\end{array}$ & $\begin{array}{l}\text { Sin } \\
\text { inclusión } \\
\text { de forraje }\end{array}$ & 2.7 & 2.9 \\
\hline \multirow[t]{2}{*}{ Forraje (g) } & $\mathrm{TCO}$ & 150.0 & - & 151.0 & - & 150.5 & - & $150.0^{\mathrm{a}}$ & $151.0^{\mathrm{a}}$ \\
\hline & MS & 25.1 & - & 25.2 & - & 25.1 & - & $24.1^{\mathrm{a}}$ & $25.2^{\mathrm{a}}$ \\
\hline \multirow{2}{*}{$\begin{array}{l}\text { Alimento } \\
\text { peletizado }(\mathrm{g})\end{array}$} & TCO & 105.7 & 105.4 & 112.2 & 106.3 & 108.9 & 105.8 & $105.5^{\mathrm{a}}$ & $109.3^{\mathrm{a}}$ \\
\hline & MS & 95.1 & 94.8 & 101.0 & 95.7 & 98.1 & 95.2 & $94.9^{\mathrm{a}}$ & $98.3^{\mathrm{a}}$ \\
\hline \multicolumn{2}{|c|}{ Días de consumo } & 88 & 101 & 90 & 112 & 89 & 106 & 94 & 101 \\
\hline \multicolumn{2}{|c|}{$\begin{array}{l}\text { Consumo promedio total } \\
\text { de alimento balanceado por } \\
\text { reproductora en TCO }(\mathrm{kg})\end{array}$} & $22.6^{\mathrm{a}}$ & $10.6^{\mathrm{a}}$ & $23.8^{\mathrm{a}}$ & $11.9^{\mathrm{a}}$ & $23.2^{\mathrm{a}}$ & $11.3^{\mathrm{b}}$ & $16.6^{\mathrm{a}}$ & $17.9^{\mathrm{a}}$ \\
\hline \multicolumn{2}{|c|}{$\begin{array}{l}\text { Consumo promedio total } \\
\text { por reproductora de MS (kg) }\end{array}$} & $10.6^{\mathrm{a}}$ & $9.6^{\mathrm{a}}$ & $11.4^{\mathrm{a}}$ & $10.7^{\mathrm{a}}$ & $11.0^{\mathrm{a}}$ & $10.2^{\mathrm{b}}$ & $10.1^{\mathrm{a}}$ & $11.1^{\mathrm{a}}$ \\
\hline \multirow{2}{*}{\multicolumn{2}{|c|}{$\begin{array}{l}\text { Consumo promedio por } \\
\text { reproductora / día de MS (g) }\end{array}$}} & $120.2^{\mathrm{a}}$ & $94.8^{\mathrm{a}}$ & $126.2^{\mathrm{a}}$ & $95.7^{\mathrm{a}}$ & $123.2^{\mathrm{a}}$ & $95.2^{\mathrm{b}}$ & $107.5^{\mathrm{a}}$ & $110.9^{a}$ \\
\hline & & \multicolumn{6}{|c|}{ Probabilidad } & & \\
\hline \multicolumn{2}{|l|}{ Energía } & \multicolumn{8}{|c|}{0.267} \\
\hline \multicolumn{2}{|l|}{ Sistema } & \multicolumn{8}{|c|}{$<0.0001$} \\
\hline \multicolumn{2}{|c|}{ Energía*Sistema } & \multicolumn{8}{|c|}{0.392} \\
\hline
\end{tabular}

${ }^{a, b}$ Letras diferentes dentro de filas indican diferencia estadística $(p<0.05)$

T1 y T2, 2.7 Mcal de energía digestible/kg de alimento balanceado con y sin inclusión de forraje verde, respectivamente; T3 y T4, $2.9 \mathrm{Mcal}$ de energía digestible/kg de alimento balanceado con y sin inclusión de forraje verde

En programas de alimentación con cuyes hembras en crecimiento, Enciso (2010) observó un mayor consumo de MS en la opción con inclusión de forraje ( $2252 \mathrm{~g}$ de consumo total), el cual consideraba un alimento de inicio con 3.0 Mcal de ED/kg de la semana 3 a la 4 y un alimento de crecimiento con $2.8 \mathrm{Mcal}$ de ED/kg de la semana 5 a la 10 , además de forraje maíz chala al $10 \%$ del peso vivo en ambos periodos; concluyendo que a bajos niveles de energía el consumo de MS tiende a incrementar, aspecto que no ocurrió en el presente estudio (Cuadro 5).

En términos de fertilidad no se hallaron diferencias estadísticas para los efectos independientes ni para la interacción de los factores (Cuadro 6). Los resultados obtenidos siguen una tendencia similar a los reportados en hembras de primer parto. Así, Dulanto (1999) obtuvo $100 \%$ de fertilidad en cuyes de las líneas Perú y Andina alimentados con dietas mixtas. Por su parte, Sarmiento (2014), trabajando con hembras gestantes bajo un sistema de alimentación integral y otro de alimentación mixta también obtuvo $100 \%$ de fertilidad en los dos tratamientos, mientras que Sarria (2011) menciona valores mínimos de $90 \%$ de fertilidad como aceptables. Por otro lado, es importante indicar que el aporte de fibra en la dieta tiene un efecto marcado en las características de las heces. Jiménez (2007) manifiesta que el aporte adecuado de fibra en la dieta evita problemas de empastamiento a nivel cecal. 
Cuadro 6. Efecto de los sistemas de alimentación y nivel de energía sobre el porcentaje de mortalidad, fertilidad, abortos, partos, tamaño de camada y peso de las crías al nacimiento y destete, mortalidad de crías nacidas y destetada en cuyes

\begin{tabular}{|c|c|c|c|c|c|c|c|c|c|c|}
\hline 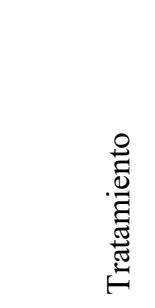 & 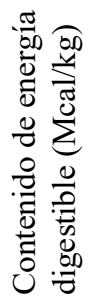 & 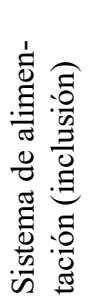 & 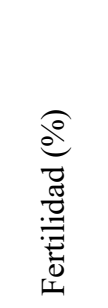 & $\begin{array}{l}\frac{0}{0} \\
\text { do } \\
\text { 융 } \\
\frac{8}{2}\end{array}$ & 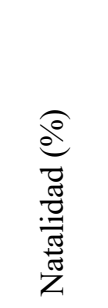 & 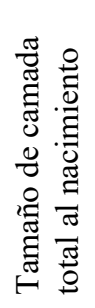 &  &  & 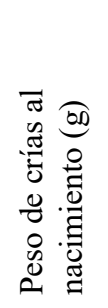 & 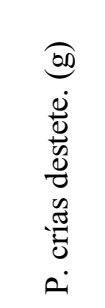 \\
\hline $\mathrm{T}-1$ & 2.70 & Con & $93.3^{\mathrm{a}}$ & - & $100.0^{\mathrm{a}}$ & $3.6^{\mathrm{a}}$ & $3.2^{\mathrm{a}}$ & $2.8^{\mathrm{a}}$ & $166^{\mathrm{a}}$ & $322^{a}$ \\
\hline $\mathrm{T}-2$ & 2.70 & $\operatorname{Sin}$ & $80.0^{\mathrm{a}}$ & $6.7^{\mathrm{a}}$ & $86.7^{\mathrm{a}}$ & $3.1^{\mathrm{a}}$ & $2.7^{\mathrm{a}}$ & $2.5^{\mathrm{a}}$ & $158^{\mathrm{a}}$ & $334^{\mathrm{a}}$ \\
\hline $\mathrm{T}-3$ & 2.90 & Con & $100.0^{\mathrm{a}}$ & - & $100.0^{\mathrm{a}}$ & $3.5^{\mathrm{a}}$ & $3.2^{\mathrm{a}}$ & $2.9^{\mathrm{a}}$ & $165^{\mathrm{a}}$ & $310^{\mathrm{a}}$ \\
\hline $\mathrm{T}-4$ & 2.90 & Sin & $80.0^{\mathrm{a}}$ & - & $100.0^{\mathrm{a}}$ & $3.2^{\mathrm{a}}$ & $2.8^{\mathrm{a}}$ & $2.6^{\mathrm{a}}$ & $157^{\mathrm{a}}$ & $339^{a}$ \\
\hline \multirow{2}{*}{\multicolumn{2}{|c|}{$\begin{array}{l}\text { Efecto del nivel de } \\
\text { energía }\end{array}$}} & 2.70 & $86.7^{\mathrm{a}}$ & $3.4^{\mathrm{a}}$ & $93.3^{\mathrm{a}}$ & $3.4^{\mathrm{a}}$ & $3.0^{\mathrm{a}}$ & $2.7^{\mathrm{a}}$ & $162.8^{\mathrm{a}}$ & $328.2^{\mathrm{a}}$ \\
\hline & & 2.90 & $91.4^{\mathrm{a}}$ & - & $100.0^{\mathrm{a}}$ & $3.4^{\mathrm{a}}$ & $3.0^{\mathrm{a}}$ & $2.8^{\mathrm{a}}$ & $161.1^{\mathrm{a}}$ & $324.7^{\mathrm{a}}$ \\
\hline \multirow{2}{*}{\multicolumn{2}{|c|}{$\begin{array}{l}\text { Efecto del sistema de } \\
\text { alimentación }\end{array}$}} & Con & $96.7^{\mathrm{a}}$ & - & $100.0^{\mathrm{a}}$ & $3.6^{\mathrm{a}}$ & $3.2^{\mathrm{a}}$ & $2.9^{\mathrm{a}}$ & $166.3^{\mathrm{a}}$ & $316.0^{\mathrm{a}}$ \\
\hline & & Sin & $80.0^{\mathrm{a}}$ & $3.4^{\mathrm{a}}$ & $93.3^{\mathrm{a}}$ & $3.2^{\mathrm{a}}$ & $2.8^{\mathrm{a}}$ & $2.6^{\mathrm{a}}$ & $157.5^{\mathrm{a}}$ & $336.8^{\mathrm{a}}$ \\
\hline \multicolumn{3}{|l|}{ Energía } & 0.647 & 0.347 & 0.081 & 0.856 & 0.733 & 0.733 & 0.773 & 0.786 \\
\hline \multicolumn{3}{|l|}{ Sistema } & 0.096 & 0.347 & 0.081 & 0.130 & 0.177 & 0.177 & 0.189 & 0.134 \\
\hline \multicolumn{3}{|c|}{ Energía*Sistema } & 0.647 & 0.347 & 0.081 & 0.598 & 0.945 & 0.945 & 0.896 & 0.515 \\
\hline
\end{tabular}

a,b Letras diferentes dentro de filas indican diferencia estadística $(p<0.05)$

T1 y T2, 2.7 Mcal de energía digestible/kg de alimento balanceado con y sin inclusión de forraje verde, respectivamente; T3 y T4, 2.9 Mcal de energía digestible/kg de alimento balanceado con y sin inclusión de forraje verde

Cuadro 7. Costo total de alimentación por cuy hembra (S/.) bajo dos sistemas de alimentación y de energía digestible

\begin{tabular}{|c|c|c|c|c|c|}
\hline \multicolumn{2}{|l|}{ Tratamiento } & $\mathrm{T} 1$ & T3 & $\mathrm{T} 2$ & $\mathrm{~T} 4$ \\
\hline \multicolumn{2}{|c|}{ Sistema de alimentación } & \multicolumn{2}{|c|}{ Con inclusión } & \multicolumn{2}{|c|}{ Sin inclusión } \\
\hline \multicolumn{2}{|c|}{ Nivel de energía (Mcal/kg) } & 2.7 & 2.9 & 2.7 & 2.9 \\
\hline \multirow{2}{*}{$\begin{array}{l}\text { Forraje } \\
\text { (chala) }\end{array}$} & Cantidad (kg) * & 13.2 & 13.6 & - & - \\
\hline & Costo/kg (S/.) & 0.16 & 0.16 & - & - \\
\hline \multirow{2}{*}{ Concentrado } & Cantidad (kg) & 9.3 & 10.1 & 10.6 & 11.9 \\
\hline & Costo $(\mathrm{S} / .)^{* *}$ & 1.11 & 1.35 & 1.13 & 1.39 \\
\hline \multicolumn{2}{|c|}{ Costo total por reproductora $(\mathrm{S} /)}$. & 12.5 & 15.8 & 12.0 & 16.5 \\
\hline \multicolumn{2}{|c|}{$\begin{array}{l}\text { Costo promedio según sistema de } \\
\text { alimentación }(\mathrm{S} /)^{1}\end{array}$} & \multicolumn{2}{|c|}{14.2} & \multicolumn{2}{|c|}{14.3} \\
\hline
\end{tabular}

T1 y T2, 2.7 Mcal de energía digestible/kg de alimento balanceado con y sin inclusión de forraje verde, respectivamente; T3 y T4, 2.9 Mcal de energía digestible/kg de alimento balanceado con y sin inclusión de forraje verde

${ }^{1}$ Tipo de cambio: S/ 2.82 por US dólar 
Cuadro 8. Costo de alimentación por cuy destetado ( $\mathrm{S} /$. ) bajo dos sistemas de alimentación y de energía digestible

\begin{tabular}{|c|c|c|c|c|}
\hline Tratamiento & $\mathrm{T} 1$ & $\mathrm{~T} 3$ & $\mathrm{~T} 2$ & $\mathrm{~T} 4$ \\
\hline Sistema de alimentación & \multicolumn{2}{|c|}{ Con inclusión de forraje } & \multicolumn{2}{|c|}{ Sin inclusión de forraje } \\
\hline Nivel de energía & 2.7 & 2.9 & 2.7 & 2.9 \\
\hline Total de hembras evaluadas & 14 & 15 & 11 & 12 \\
\hline Crías destetadas totales & 39 & 43 & 28 & 31 \\
\hline Promedio de camada al destete & 2.8 & 2.9 & 2.5 & 2.6 \\
\hline Costo de reproductora (S/.) & 12.50 & 15.80 & 12.00 & 16.50 \\
\hline Costo total $(\mathrm{S} /)$. & 4.50 & 5.40 & 4.80 & 6.30 \\
\hline $\begin{array}{l}\text { Costo promedio por cuy destetado } \\
\text { por sistema de alimentación }\left(\mathrm{S} / \mathrm{s}^{1}\right.\end{array}$ & \multicolumn{2}{|c|}{5.00} & \multicolumn{2}{|c|}{5.60} \\
\hline
\end{tabular}

T1 y T2, 2.7 Mcal de energía digestible/kg de alimento balanceado con y sin inclusión de forraje verde, respectivamente; T3 y T4, 2.9 Mcal de energía digestible/kg de alimento balanceado con y sin inclusión de forraje verde

${ }^{1}$ Tipo de cambio: S/ 2.82 por US dólar

El tamaño de camada al nacimiento fue similar en todos los tratamientos (Cuadro 6), al igual que ocurrió en el experimento de Solórzano (2014). Por otro lado, Revilla (2011), bajo suplementación al 30\% de minerales orgánicos por debajo del requerimiento del NRC (1995), logró mayor tamaño de camada (3.3) para cuyes en sistemas de alimentación integral, en comparación con el tamaño de camada de 2.8 crías del presente estudio.

No se encontraron diferencias significativas en el peso al destete entre tratamientos para los efectos individuales ni en la interacción de estos. Estos resultados discrepan del estudio de Solórzano (2014) quien encontró un mayor peso promedio con dietas sin inclusión de forraje. Por otro lado, los resultados de Dulanto (1999) con cuyes de la línea Perú son similares a los obtenidos en el T1 y T3 en sistemas de alimentación con inclusión de forraje, pero inferiores a los reportados por Revilla (2011) y Pedraz (2001) a los 14 días de lactancia.

La mortalidad fue escasa (cuatro muertos en los sistemas sin inclusión y dos en sis- temas con inclusión de forraje) y solo se registró en el último tercio de gestación, con cuadros neumónicos, torsión uterina en un caso con alto número de crías, y solo dos incidencias sugeridas a $E$. coli, en dietas sin inclusión de forraje. Revilla (2011) sugiere usar premezclas que aporten el 30\% adicional del requerimiento de minerales recomendado por el NRC (1995). Así mismo, Vergara (2008) y Sarria (2011) indican que la inclusión de forraje en la dieta permite cubrir algunas falencias en la formulación de balanceados integrales o únicos.

Con relación al análisis económico, se pudo determinar que el factor que incrementa el costo en la alimentación de las reproductoras es el nivel de energía, debido a un adicional de insumos como maíz amarillo y aceite vegetal; mientras que a nivel de sistemas con y sin forraje, se igualan los costos por madre (Cuadro 7).

Revilla (2011) en dietas isoenergéticas de $2.9 \mathrm{Mcal}$ de ED/kg obtuvo S/. 12.20 de costo por reproductora evaluada, mientras que el presente estudio se alcanzó el valor de $\mathrm{S} / .14 .30$ para $\mathrm{T} 4$ en similares condiciones 
(nivel de energía y sistema de alimentación). Estos resultados expuestos difieren del estudio de Solórzano (2014) quien obtuvo costos más altos para sus dietas integrales (S/. 15.90 por reproductora), pero en 18 semanas de evaluación.

El costo obtenido por cada cuy destetado (Cuadro 8) denota que los costos más elevados se obtuvieron bajo el sistema de alimentación integral; valor que se vio afectado por al menor tamaño de camada. Por otro lado, las dietas con mayor nivel de energía tienen costos más altos de producción. Revilla (2011) y Solórzano (2014) concuerdan que los sistemas con inclusión de forraje en zonas forrajeras presentan costos más bajos para madres y crías producidas. El factor económico marca la diferencia entre tratamientos, permitiendo la toma de decisiones para llevar con éxito la actividad empresarial (Sarria, 2011). En el presente estudio, a pesar de no estar en un entorno agrícola, se observa que la inclusión de forraje verde no afectó el costo de producción; sino por el contrario, permitió tener menor costo por cría lograda (S/. 5.00 vs. S/. 5.60) al haber conseguido mayor tamaño de camada al destete.

\section{Conclusiones}

- Los niveles de energía digestible (2.7 y $2.9 \mathrm{Mcal}$ de ED $/ \mathrm{kg}$ ) en dietas con o sin inclusión de forraje verde no afectaron significativamente la fertilidad, tasa de abortos, natalidad, y tamaño de camada al nacimiento y al destete.

- Los sistemas con y sin exclusión de forraje, denominados comercialmente mixto e integral, no mostraron diferencias significativas en el comportamiento productivo y reproductivo general de las madres reproductoras y de las crías resultantes.

- Las reproductoras bajo sistema de inclusión de forraje perdieron peso al destete $(-47.6 \mathrm{~g})$, mientras que dietas con el nivel de energía $2.9 \mathrm{Mcal}$ de $\mathrm{ED} / \mathrm{kg}$ de alimento produjeron mayor peso al parto $(\mathrm{p}<0.05)$.
- El consumo total de alimento en materia seca de las reproductoras fue mayor $(p<0.01)$ en el sistema de alimentación con inclusión de forraje verde.

- Los costos de alimentación fueron más altos en dietas que tenían el mayor nivel de energía probado. El menor costo por cría destetada se obtuvo bajo el sistema de alimentación con inclusión de forraje ( $\mathrm{S} / 5.00$ vs. S/.5.60), así como en el menor nivel de energía (S/. 4.65 vs. S/. 5.85).

\section{Literatura Citada}

1. Airahuacho F, Vergara V. 2017. Evaluación de dos niveles de energía digestible en base a los estándares nutricionales del NRC (1995) en dietas de crecimiento para cuyes (Cavia porcellus L). Rev Inv Vet Perú 28: 255 264. doi: 10.15381/rivep.v28i2.13079

2. AOAC International. 2005. Official methods of analysis of AOAC International. $18^{\text {th }}$ ed. Gaithersburg, USA: AOAC International. $179 \mathrm{p}$.

3. Camino J, Hidalgo V. 2014. Evaluación de dos genotipos de cuyes (Cavia porcellus) alimentados con concentrado y exclusión de forraje verde. Rev Inv Vet Perú 25: 190-197. doi: /10.15381/ rivep.v25i2.8490

4. Chauca L, Remigio R, Valverde N, Vergara V. 2008. Evaluación de raciones para cuyes (Cavia porcellus) reproductoras y lactantes de las razas Perú y cruzados. En: XXXI Reunión Científica Anual de la Asociación Peruana de Producción Animal. Lima, Perú.

5. Condori R. 2014. Evaluación de bajos niveles de fibra en dietas de inicio y crecimiento de cuyes (Cavia porcellus) con exclusión de forraje. Tesis de Ingeniero Zootecnista. Lima: Univ. Nacional Agraria La Molina. $77 \mathrm{p}$.

6. Correa R. 1988. La crianza del cuy. Pasto, Colombia: Instituto Colombiano Agropecuario. $47 \mathrm{p}$. 
7. Dulanto M. 1999. Parámetros productivos y reproductivos de tres líneas puras y 2 grados de cruzamiento entre líneas de cuyes (Cavia porcellus). Tesis de Ingeniero Zootecnista. Lima: Univ. Nacional Agraria La Molina. 92 p.

8. Enciso P. 2010. Evaluación de cinco programas de alimentación sobre el inicio de la pubertad en cuyes (Cavia porcellus) raza Andina. Tesis de Magíster. Lima: Univ. Nacional Agraria La Molina. 108 p.

9. Jiménez Y. 2007. Valoración energética de diferentes tipos de maíz (Zea mays) utilizado en la alimentación de cuyes (Cavia porcellus). Tesis de Ingeniero Zootecnista. Riobamba. Ecuador: Escuela Superior Politécnica de Chimborazo. $60 \mathrm{p}$.

10. Morales A, Carcelén F, Ara M, Arbaiza T, Chauca L. 2011. Evaluación de dos niveles de energía en el comportamiento productivo de cuyes (Cavia porcellus) de la raza Perú. Rev Inv Vet Perú 22: 177-182. doi: 10.15381/ rivep.v22i3.254

11. [NRC] National Research Council. 1978. Nutrient requirements of the guinea pig. In: Nutrient requirements of laboratory animals. $3^{\text {rd }}$ Revised Ed. Washington DC, USA: National Academy Press. p 59-59.

12. [NRC] National Research Council. 1995. Nutrient requirements of laboratory animals: guinea pig. Washington, DC National Academy Press. USA: NRC. $192 \mathrm{p}$.

13. Pedraz F. 2001. Evaluación reproductiva de cuyes mejorados (Cavia porcellus) procedentes de Arequipa, Cajamarca y Lima. Tesis de Ingeniero Zootecnista. Lima: Univ. Nacional Agraria La Molina. 95 p.
14. Peruano D. 1999. Evaluación de la vida productiva y reproductiva del cuy hembra (Cavia porcellus) en cuatro partos utilizando empadre continuo. Tesis de Ingeniero Zootecnista. Lima: Univ. Nacional Agraria La Molina. $88 \mathrm{p}$.

15. Remigio R. 2006. Evaluación de tres niveles de lisina y aminoácidos azufrados en dietas de crecimiento para cuyes (Cavia porcellus L) mejorados. Tesis de Magíster. Lima, Perú: Univ. Nacional Agraria La Molina. $97 \mathrm{p}$.

16. Revilla J. 2011. Evaluación de la performance de cuyes (Cavia porcellus) suplementados con minerales orgánicos quelados en la fase de producción. Tesis de Ingeniero Zootecnista. Lima: Univ. Nacional Agraria La Molina. $108 \mathrm{p}$.

17. Sarmiento J. 2014. Diferentes niveles de vitamina $C$ sobre el comportamiento productivo del cuy (Cavia porcellus) hembra bajo alimentación integral. Tesis de Ingeniero Zootecnista. Lima: Univ. Nacional Agraria La Molina.

18. Sarria, J. 2011. El cuy crianza tecnificada. Manual técnico en cuyicultura $\mathrm{N}^{\mathrm{o}} 1$. Oficina Académica de Extensión y Proyección Social. Universidad Nacional Agraria La Molina. Lima, Perú. 43 p.

19. Solórzano J. 2014. Evaluación de tres sistemas de alimentación comercial de cuyes (Cavia porcellus) en la etapa de reproducción. Tesis de Ingeniero Zootecnista. Lima: Univ. Nacional Agraria La Molina. $124 \mathrm{p}$.

20. Torres A, Chauca L, Vergara V. 2006. Evaluación de dos niveles de energía y proteína en dietas de crecimiento y engorde de cuyes machos. En: XXIX Reunión de la Asociación Peruana de Producción Animal. Huancayo: APPA.

21. Vergara V. 2008. Avances en nutrición y alimentación en cuyes. En: XXXI Reunión Científica Anual de la Asociación Peruana de Producción Animal. Lima, Perú. 